\title{
Modelling of simple shear tests on volcanic unsaturated sands
}

\author{
Mariagiovanna Moscariello, ${ }^{1, *}$, Yanni Chen ${ }^{2}$, Sabatino Cuomo ${ }^{1}$, and Giuseppe Buscarnera ${ }^{2}$ \\ ${ }^{1}$ University of Salerno, Lab. Geotechnics, Department of Civil Engineering, Salerno, Italy \\ ${ }^{2}$ Northwestern University, Dept. of Civil and Environmental Engineering, Evanston, USA.
}

\begin{abstract}
In landslide susceptibility analysis, a relevant issue is the proper modelling of the complex mechanisms that regulate the failure and post-failure stages. In this paper, simple shear experiments replicating the kinematics of failure in landslide-prone areas are interpreted through an elastoplastic strain-hardening constitutive model for both saturated and unsaturated soils. The material tested is an air-fall volcanic (pyroclastic) soil from Southern Italy which originated from the explosive activity of the Somma-Vesuvius volcanic apparatus. Data from triaxial and shear tests performed on remoulded specimens characterized by saturated and unsaturated conditions are used to calibrate the model parameters. The evolution of shear stress, volumetric and shear strain measured during the experiments are reproduced by means of a model formulation specific for simple shear conditions. To capture the strength emerging under different states of saturation, non-associated flow rule, and a suction-dependent yield surface are used. Examination of the experimental data available for various testing conditions enabled the quantification of the variability of fundamental model constants, such as those controlling frictional resistance and water retention behaviour. To account for such scatter in the physical properties, the constitutive analyses are performed by employing varying model constants within a band of admissible values. The resulting model performance is validated by comparing the simulations with the experimental results at different saturation conditions. The results show that the combination of the proposed model with a data-driven determination of the range of variation of hydro-mechanical properties is crucial to satisfactorily simulate the essential features of the soil response under a variety of simple shear testing regimes.
\end{abstract}

\section{Introduction}

The occurrence of rainfall-induced shallow landslides of the flow-type has posed great threats to densely populated regions due to its long-travel distance and high runout velocity [1]. Massive events have been recorded in unsaturated pyroclastic deposits in Southern Italy, where the activities of Somma-Vesuvius volcanic system have produced vast amounts of pyroclastic soil along steep slopes. Upon wetting, the suction drop within the unsaturated regime weakens the soil, thus increasing its potential to experience shear failure and move downslope through a rapid flow process [2]. For this reason, experimental and numerical tools are highly needed to identify the material properties responsible for such dramatic failures, as well as to estimate the regional landslides susceptibility [3, 4].

Recently, a range of laboratory experiments based on triaxial and shear configurations have been carried out on samples obtained from the Sarno-Quindici area to clarify the mechanical characteristics of the pyroclastic soils. Specifically, the simple shear devices have been improved to extend their use to partially saturated soils [5] and multiple loading sequences have been investigated to mimic the in-situ conditions of the shallow slopes. The pyroclastic soils were generally categorized as coarse-grained materials and tested under both saturated and unsaturated conditions. The simple shear tests on saturated samples

*e-mail: mamoscariello@unisa.it normally experience the contractive processes, while on unsaturated samples display a transition from contractive to dilative response [6].

Quantifying such transitions, as well as their role in triggering flow instabilities, requires effective constitutive relationships able to cope with a varying degree of saturation. This paper discusses a modeling strategy aimed at combining such recent experimental data with an advanced constitutive model for pyroclastic soils. Specifically, the following sections make reference to simple shear testing conditions, with the purpose to constrain the range of admissible model constants leading to accurate numerical simulations under both saturated and unsaturated regimes. Special attention is given to the variation of the soil properties and its effect on the model performance by allowing the parameters to vary within a range defined from the scatter of the experimental data. For simplicity purpose, the scope of the analysis has been restricted to the parameters that control the frictional resistance, and those describing the soil water retention curve (SWRC), with the ultimate goal to ascertain whether such simple steps are sufficient to lead to satisfactory simulations.

\section{Constitutive model}

Due to the multiphase interactions among the solid, fluid, and gas phases constituting an unsaturated soil, quantifying the stress state acting on the solid skeleton requires 
special treatments. In this context, a particular stress measure often used to quantify the skeleton stress is the socalled Bishop effective stress [7-9], expressed as follows:

$$
\sigma_{i j}^{\prime}=\sigma_{i j}-u_{\mathrm{a}} \delta_{i j}+S_{r} s \delta_{i j}
$$

where $S_{r}$ and $s$ are degree of saturation and suction, respectively, while $\sigma_{i j}$ is the total stress, $u_{\mathrm{a}}$ is the pore air pressure, usually assumed constant and equal to the atmospheric pressure, and $\delta_{i j}$ is the Kronecker delta. The relationship between $S_{r}$ and $s$ is defined by the SWRC and generally interpreted with the van Genuchten retention model [10]:

$$
\theta(s)=\theta_{r}+\frac{\left(\theta_{s}-\theta_{r}\right)}{\left[1+\left(\alpha_{v g} s\right)^{n_{v g}}\right]^{m_{v g}}}
$$

where $\theta$ is the volumetric water content, $\theta_{r}$ and $\theta_{\mathrm{s}}$ are its values at residual and saturated conditions, and $\alpha_{v g}, n_{v g}$ and $m_{v g}=1-1 / n_{v g}$ are shape parameters.

For coarse-grain materials like pyroclastic soils, a frictional relation is often used to define the yielding conditions [11]:

$$
f=\tau-\eta_{Y} \sigma^{\prime}
$$

where $\eta_{Y}$ is the stress ratio at yielding, $\tau$ is the shear stress and $\sigma^{\prime}$ is the effective normal stress. And as indicated experimentally, the unsaturated samples have the possibility to experience the transition from contractive to dilative responses. For this reason, to account for the role of density on the volume change of variably saturated pyroclastic soil samples, the concept of state parameter $\psi[12,13]$ is introduced into a non-associated flow rule by adjusting the dilatancy function proposed by Lagioia et. al. [14], as follow:

$$
\begin{aligned}
D=\frac{\mathrm{d} \varepsilon_{p}}{\mathrm{~d} \gamma_{p}} & =\mu_{g}\left(M_{g}^{*}-\eta\right)\left(\frac{\alpha_{g} M_{g}^{*}}{\eta}+1\right) \\
M_{g}^{*} & =M_{g} \exp \left(m_{g} \psi\right)
\end{aligned}
$$

where $\mu_{g}, m_{g}$, and $\alpha_{g}$ are shape parameters and $M_{g}$ is the critical stress ratio governed by the friction angle $\varphi$ (i.e., $\left.M_{g}=\tan (\varphi)\right)$. In the above equation, the state parameter $\psi$ is defined as the difference between the current porosity $n$ and the critical state porosity $n_{c s}$. Specifically, since the state parameter $\psi$ quantifies the distance between current state and the critical state line (CSL) in the $n-\log \sigma^{\prime}$ space, its introduction into the constitutive relationship makes the model porosity-dependent. Furthermore, since evidence available in the literature suggests that suction causes an upward shift the CSL [15], the state parameter can be augmented by incorporating suction into its definition, as follows:

$$
\psi=n-n_{c s}(1+b s), \quad n_{c s}=N_{c s}-\lambda_{c s} \ln \sigma^{\prime}
$$

where $N_{c s}$ and $\lambda_{c s}$ represent the intercept and slope of the CSL in the $n-\log \sigma^{\prime}$ space corresponding to fully saturation, and $b$ reflects the effects of $s$ on the CSL. $\varepsilon$ and $\gamma$ are the normal and shear strains, respectively, with the superscript $p$ representing their plastic portions, computed as:

$$
\mathrm{d} \varepsilon^{p}=\Lambda \frac{D}{\sqrt{1+D^{2}}}, \quad \mathrm{~d} \gamma^{p}=\Lambda \frac{1}{\sqrt{1+D^{2}}}
$$

where $\Lambda$ is the plastic multiplier derived from the consistency condition. The hardening law is defined as:

$$
\mathrm{d} \eta_{Y}=\left(M_{p}-\eta_{Y}\right) \frac{1}{\lambda} \mathrm{d} \gamma_{p}
$$

where $\lambda$ is a hardening constant and $M_{\mathrm{p}}=M_{\mathrm{g}}$ for simplicity. Furthermore, the elastic responses are captured by hypoelasticity with the Young's modulus $E$ and shear modulus $G$ expressed as:

$$
E=E_{r}\left(\frac{\sigma^{\prime}}{\sigma_{r}}\right)^{n_{E}}, \quad G=G_{r}\left(\frac{\sigma^{\prime}}{\sigma_{r}}\right)^{n_{G}}
$$

where $n_{E}$ and $n_{G}$ are constant power law coefficients; $E_{\mathrm{r}}$ and $G_{\mathrm{r}}$ are the values of the elastic moduli at the reference stress $\sigma_{\mathrm{r}}$ (normally being $1 \mathrm{kPa}$ ).

\section{Calibration procedure}

The model parameters were calibrated based on the experimental data available for the pyroclastic soil derived from the explosive activity of the Somma-Vesuvius volcanic apparatus (Southern Italy). The experimental data on remolded specimens are used to illustrate the model calibration procedure.

Parameters related to the SWRC were calibrated using two wetting tests under simple shear conditions. The tests were performed at constant normal stress (30 and $50 \mathrm{kPa}$ ) and constant shear stress (34 and $51 \mathrm{kPa}$ ), and the suction was zeroing with a fixed rate of $0.1 \mathrm{kPa} / \mathrm{h}$. The SWRCs are extracted from the wetting tests considering only the part at constant and low shear strain $(\gamma \approx 0.05)$. Figure 1 illustrates the experimental data along with the calibrated van Genuchten retention model. The figure also illustrates the estimated upper and lower bounds of the water retention curve, here defined to encompass the $95 \%$ confidence

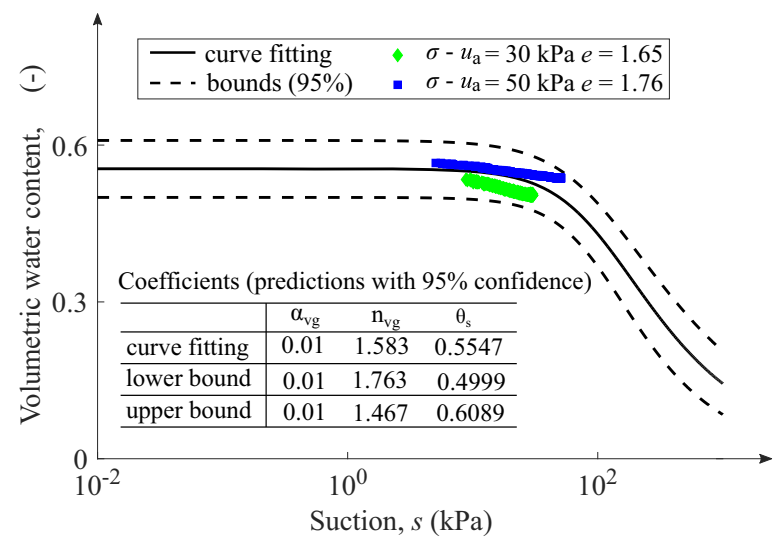

Figure 1. Calibration of the SWRCs interpolated using the van Genuchten model. Dashed lines represent the prediction bounds for a new observation with $95 \%$ confidence level. And the table lists the values of the fitting coefficients with $\theta_{r}=0.0002$. 


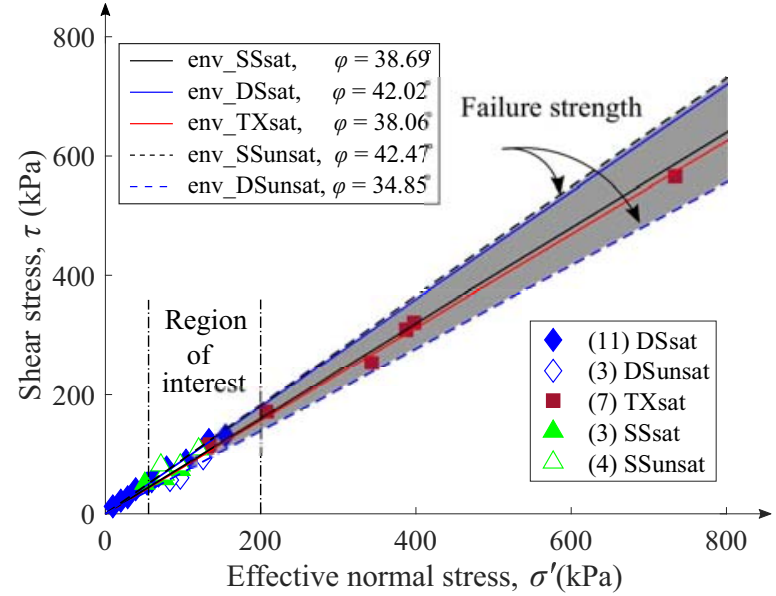

Figure 2. Shear strength envelope determined through Simple Shear tests (SS), Direct Shear tests (DS) and Triaxial tests (TX) on saturated and unsaturated specimens (SS, TX and DS data is taken from [16], [17] and [18]). The numbers in the brackets are the number of tests considered to obtain shear strength envelopes; the region of interest represents the range of effective stress investigated using numerical simulation.

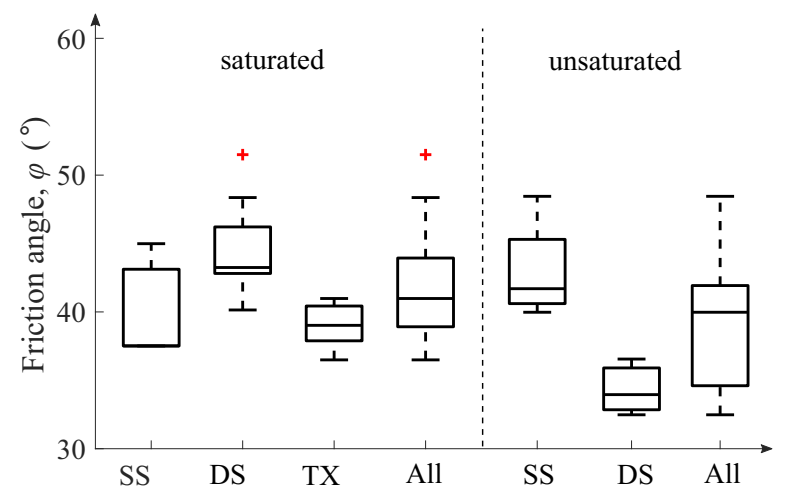

Figure 3. Variability of $\varphi$ under various testing protocols.

interval associated with the available data (dashed lines in Figure 1). The fitting coefficients $\left(\alpha_{v g}, \theta_{s}\right.$ and $n_{v g}$ with $\left.\theta_{r}=2 e^{-5}\right)$ are reported in Figure 1.

The critical stress ratio $M_{g}$ is calibrated on the basis of the results obtained with three different testing devices, including Direct Shear (DS), Simple Shear (SS) and Triaxial (TX) apparatus, under various saturation conditions, as shown in Figure 2 [16]. The list of tests considered is reported in Table 1. Five shear strength envelopes specified for different testing conditions are obtained through a linear fitting with no cohesion and the friction angle is evaluated for each test as $\varphi=\arctan \left(\tau / \sigma^{\prime}\right)$. The results readily illustrate the variability of the friction angle $\varphi$ under different testing conditions. To further illustrate the variability of $\varphi$, Figure 3 describes the distribution of $\varphi$ for each of the imposed testing regimes through maximum, minimum, mean first and fourth quantile. Comparisons between saturated and unsaturated samples indicate that the presence of suction is responsible for an additional variation in the friction properties. Therefore, a relationship between friction

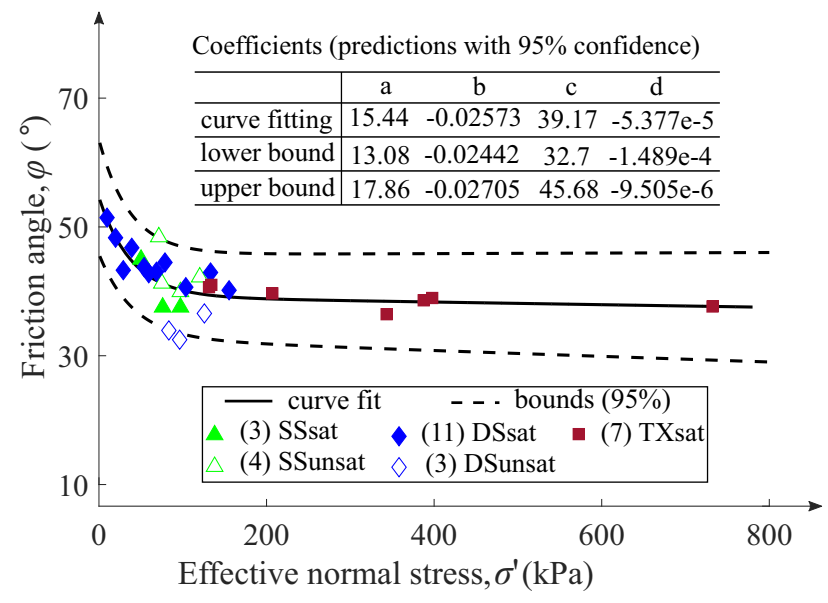

Figure 4. Relationship between friction angle and effective normal stress. Dashed lines represent the prediction bounds associated to $95 \%$ confidence level.

angle $(\varphi)$ and effective normal stress $\left(\sigma^{\prime}\right)$ is established to account for the stress-dependent failure strength as represented in Figure 4. An exponential expression is used to obtain the best fit between $\sigma^{\prime}$ and $\varphi$, which is expressed as:

$$
\varphi \sigma^{\prime}=a \cdot \exp b \cdot \sigma^{\prime}+c \cdot \exp d \cdot \sigma^{\prime}
$$

where $a, b, c$, and $d$ are fitting parameters and their values are listed in Fig. 4. Despite the good agreement with the measured trends illustrated in Fig. 4, the large data scatter resulted into an $R^{2}=0.51$. Thus, similar to the variability in SWRC, the prediction bounds for a new observation with $95 \%$ confidence level are also provided in Figure 4 to quantify the uncertainty in estimating $\varphi$ and the fitting coefficients are summarized in Figure 4. In the following sections, their role will be studied with reference to simple shear tests conducted under both saturated and unsaturated conditions.

The critical state parameters $N_{\mathrm{cs}}$ and $\lambda_{\mathrm{cs}}$ have been constrained in the $n-\log \sigma^{\prime}$ plane. Since none of the TX, DS and SS tests fully reached the critical state, the precise determination of the location of the Critical State Line (CSL) was not possible. Hence, approximately, the CSL is evaluated by hypothesizing that the Critical State is reached at large deformations (e.g. for TX tests it was supposed that CSL was reached at axial strain equals to $40 \%$ ). Specifically, for SS tests, the CSL is defined by extrapolating the experimental results for an extra 5\% shear strain $\gamma$. As shown in Figure 5, $\lambda_{\mathrm{cs}}=0.031$ and $N_{\mathrm{cs}}=0.78$.

The model parameters characterising the dilatancy expression (Eq. 4) are calibrated based on simple shear tests on saturated and unsaturated specimens. The experimental values of dilatancy are computed as $D=\Delta \varepsilon / \Delta \gamma$ by neglecting the elastic portion of the strain. As shown in Figure 6, a series of discrete values of the measured dilatancy is computed for every $0.4 \%$ increase in shear strain $\gamma$ for a saturated sample (test ID. SSP0315) and an unsaturated sample (test ID. SSRPSG24). With a single set of dilatancy parameters being $a_{g}=0.001, m_{g}=0.99, \mu_{g}=0.2$, and $b=0.02$ and considering the variation in $\varphi$, the pro- 
Table 1. List of the test adopted to extrapolate shear strength envelope (data from [17], [16] and [18]).

\begin{tabular}{|c|c|c|c|c|c|}
\hline Test type & Condition & Test ID. & $s[\mathrm{kPa}]$ & $\sigma-u_{a}[\mathrm{kPa}]$ & $\sigma^{\prime}$ \\
\hline \multirow{7}{*}{ Simple Shear } & sat & SSP0115 & 0.0 & 100.0 & 100.0 \\
\hline & sat & SSP0315 & 0.0 & 76.0 & 76.0 \\
\hline & sat & SSP0215 & 0.0 & 50.5 & 50.5 \\
\hline & unsat & SSRPSF03a & 27.5 & 97.0 & 120.1 \\
\hline & unsat & SSRPSF03b & 14.7 & 75.4 & 138.6 \\
\hline & unsat & SSRPSG23 & 25.0 & 50.0 & 73.0 \\
\hline & unsat & SSRPSG24 & 25.0 & 100.0 & 118.9 \\
\hline \multirow{14}{*}{ Ditect Shear } & sat & TASNRS21 & 0.0 & 9.0 & 9.0 \\
\hline & sat & TASNRS22 & 0.0 & 20.0 & 20.0 \\
\hline & sat & TASNRS23 & 0.0 & 29.0 & 29.0 \\
\hline & sat & TASNRS25 & 0.0 & 39.0 & 39.0 \\
\hline & sat & TASNRS24 & 0.0 & 54.0 & 54.0 \\
\hline & sat & TASNRS26 & 0.0 & 59.0 & 59.0 \\
\hline & sat & TASNRS29 & 0.0 & 69.0 & 69.0 \\
\hline & sat & TASNRS28 & 0.0 & 79.0 & 79.0 \\
\hline & sat & TASNRS27 & 0.0 & 104.0 & 104.0 \\
\hline & sat & TASNRS31 & 0.0 & 133.0 & 133.0 \\
\hline & sat & TASNRS32 & 0.0 & 135.0 & 135.0 \\
\hline & unsat & TAL0608 & 25.0 & 73.4 & 73.4 \\
\hline & unsat & TAL0808 & 45.0 & 50.0 & 50.0 \\
\hline & unsat & TAL1008 & 60.0 & 89.3 & 89.3 \\
\hline Test type & Condition & Test ID. & $s[\mathrm{kPa}]$ & $p-u_{a}[\mathrm{kPa}]$ & $p^{\prime}$ \\
\hline \multirow{7}{*}{ Triaxial } & sat & BIS2206 & 0.0 & 100.0 & 100.0 \\
\hline & sat & BIS2306 & 0.0 & 100.0 & 100.0 \\
\hline & sat & BIS2406 & 0.0 & 100.0 & 100.0 \\
\hline & sat & BIS2606 & 0.0 & 50.0 & 50.0 \\
\hline & sat & BIS2706 & 0.0 & 200.0 & 200.0 \\
\hline & sat & BIS2806 & 0.0 & 30.0 & 30.0 \\
\hline & sat & BIS2906 & 0.0 & 30.0 & 30.0 \\
\hline
\end{tabular}

Table 2. Summary of the fixed model parameters

\begin{tabular}{cccc}
\hline Category & Symbols & Unit & Pyroclastic soil \\
\hline \multirow{5}{*}{ Elasticity } & $E_{r}$ & $\mathrm{kPa}$ & 8000 \\
& $G_{r}$ & $\mathrm{kPa}$ & 1000 \\
& $\sigma_{r}$ & $\mathrm{kPa}$ & 1.0 \\
& $n_{\mathrm{E}}$ & - & 0.5 \\
& $n_{\mathrm{G}}$ & - & 0.8 \\
\hline \multirow{5}{*}{ Plasticity } & $\alpha_{g}$ & - & 0.001 \\
& $m_{g}$ & - & 0.99 \\
& $\mu_{g}$ & - & 0.2 \\
& $b$ & $1 / \mathrm{kPa}$ & 0.02 \\
\multirow{2}{*}{ CSL } & $\lambda$ & - & 0.06 \\
& $N_{c s}$ & - & 0.78 \\
& $\lambda_{c s}$ & - & 0.031 \\
\hline
\end{tabular}

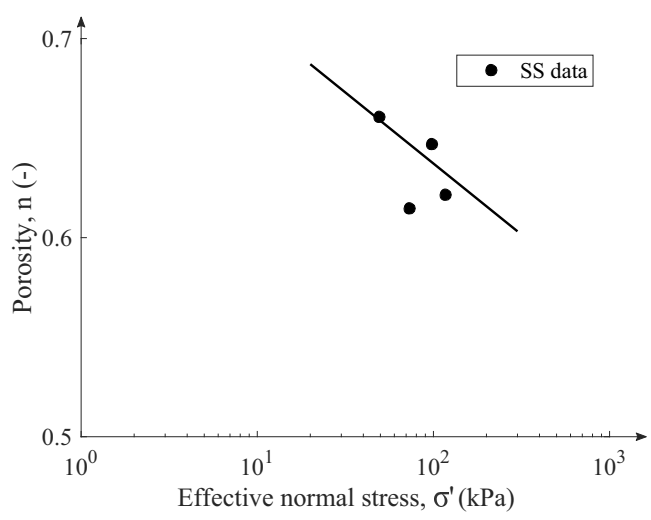

Figure 5. Critical State Line for Simple Shear tests. By fitting the experimental data, the CSL in $\log \sigma^{\prime}-n$ space is defined with $\lambda_{\mathrm{cs}}=0.031$ and $N_{\mathrm{cs}}=0.78$

posed dilatancy law in Eq. 4 can reproduce the main trends of the experimental data and capture the transition from contraction to dilation in unsaturated samples.

The elastic response has been modelled through a pressure-dependent hypoelastic model and the related parameters are calibrated by using SS tests on saturated specimens. Parameters $E_{r}, n_{\mathrm{E}}$ are evaluated in the $\sigma^{\prime}-\varepsilon$ plane while $G_{r}, n_{\mathrm{G}}$ are evaluated in the $\tau$ - $\gamma$ plane.

Finally, the hardening parameter $(\lambda)$ has been determined through a trial-and-error procedure being 0.06 .

\section{Role of the variability of pyroclastic soil properties}

As discussed above, most of the parameters can be determined according to their physical meaning, as well as with the aid of the available experimental datasets. However, the estimation of some material properties like friction angle and SWRC shows uncertainties and further investigation is needed to clarify their influence on the material response. For this reason, variability analyses are carried out to investigate the effect of the model parameters associated with frictional strength and water retention on the model performance. The fitting relationship obtained in Figure 1 and 4 is adopted to define a range constraining the upper and lower boundaries of selected material characteristics.

Figure 7 shows the model performance for two specific saturated samples (test ID. SSP0115 and SSP0315). The simulation results are represented by the shaded area formed by considering the variability in friction angle, furthermore in $M_{g}$, to reflect the uncertainty in shear strength envelope. The solid black curve represents the result from the curve fitting values, while the dashed boundaries imply the bounds associated with prediction bounds of $\varphi$ of $95 \%$ confidence level. It is readily apparent that the experimental results are well captured by the prediction bounds for the considered samples.

Similarly, the analyses are repeated for two unsaturated samples (test ID. SSRPSG23 and SSRPSG24). And since the SWRC plays an important role in unsaturated samples, therefore, in addition to the variability of $\varphi$, also the variability of the constants governing the SWRC is considered. As presented in Figure 8, the predicted responses match satisfactorily the $\gamma-\tau$ and $\gamma-S_{r}$ relationship, but display mismatches in the $\gamma-\varepsilon$ responses. However, the difference between measurements and predictions can be considered acceptable and the model is capable to properly replicate the experimental trend. Furthermore, it is worth to point out that unlike SSRPSG24, the change in the experimental results of $S_{r}$ in sample SSRPSG23 is more severe, but it is included in the shaded area (Fig. 8c). The mismatch between experimental and numerical results can be due to the difference in the imposed loading rate upon shearing. The effect of loading rate needs to be further explored in future studies. In particular, future analyses may consider the possible emergence of suction heterogeneity in the proximity of instability conditions, which can in principle lead to transients reflecting a loss of equilibration [19]. Similarly, further validation tests of the proposed calibration approach may involve the simulation of 

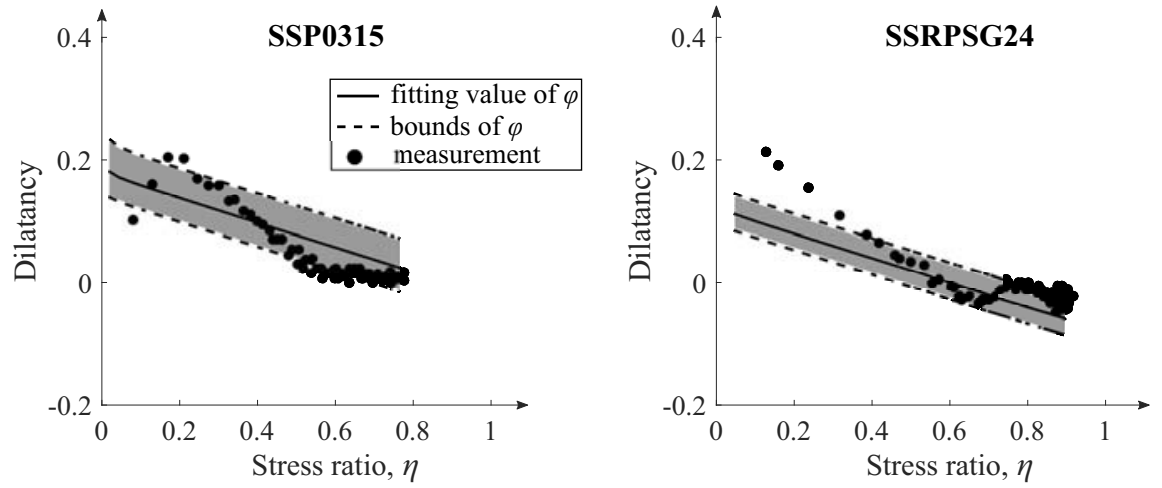

Figure 6. Calibration of dilatancy parameters with saturated and unsaturated samples: a) test ID. SSP0315; b) test ID. SSRPSG24. Model responses are obtained with $a_{g}=0.001, m_{g}=0.99, \mu_{g}=0.2$, and $b=0.02$ considering the variability in friction angle $\varphi$.
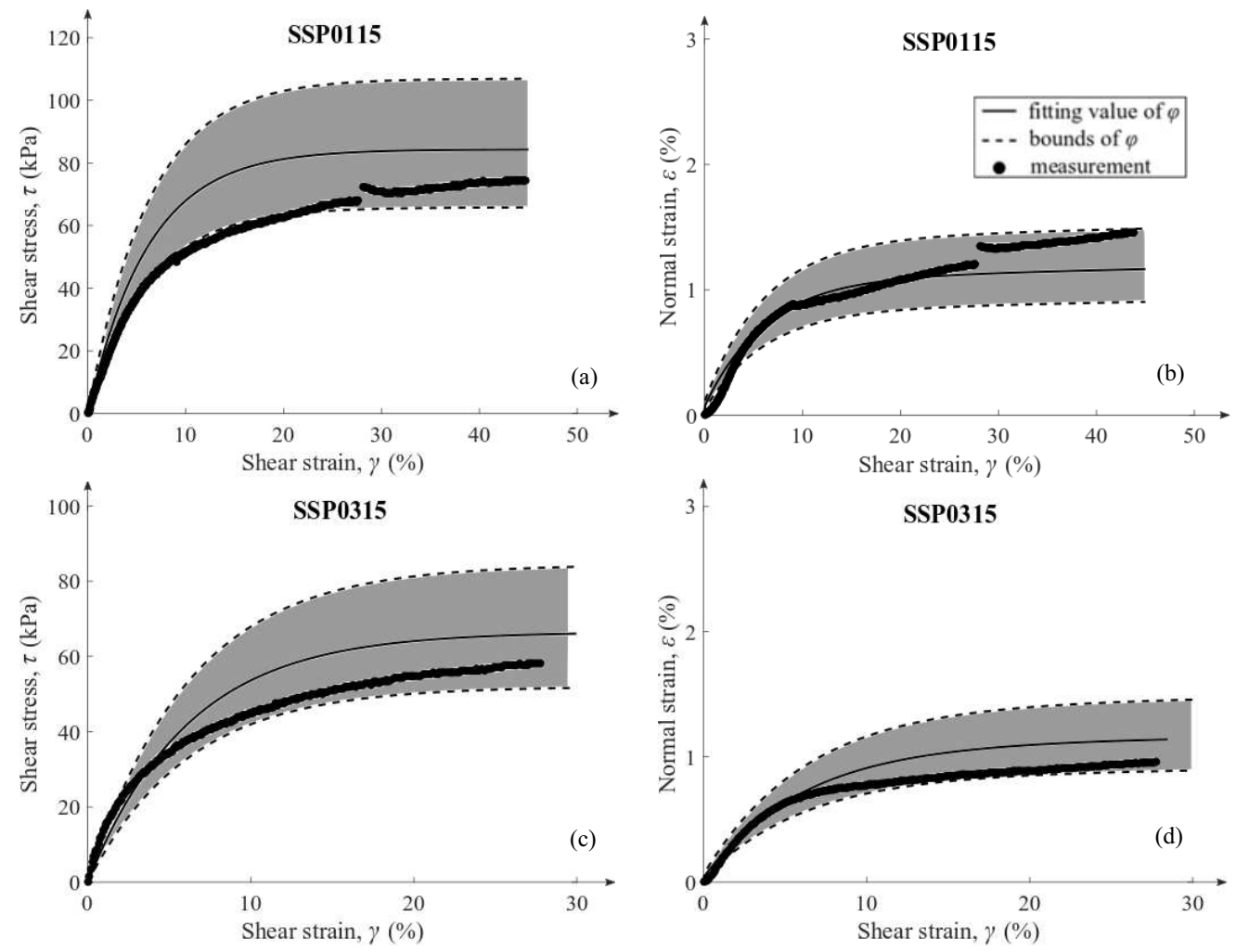

Figure 7. Simulations of SS tests under saturated conditions:(a) $\gamma-\tau$ response of test ID. SSP0115; (b) $\gamma-\varepsilon$ response of test ID. SSP0115; (c) $\gamma-\tau$ response of test ID. SSP0315; (d) $\gamma-\varepsilon$ response of test ID. SSP0315.

wetting tests, which are a known source of mechanical instabilities involving a flow-like behavior $[20,21]$.

\section{Conclusion}

The paper discusses the modelling of the simple shear tests performed on a volcanic soil sampled from Southern Italy. A constitutive model specialized for simple shear conditions has been proposed and multiple laboratory tests, including triaxial, direct and simple shear tests on saturated and unsaturated specimens, have been used to calibrate the model parameters, as well as to assess their range of variations in model and its effect on the material response. Specially, variability analyses were carried out to investigate the impacts of changes in friction angle and SWRC properties. The model performance was evaluated by comparing the resulting simulations with data from simple shear tests conducted under constant suction and examined in $\tau-\gamma$ and $\varepsilon-\gamma$ and $\gamma-S_{r}$ planes. The results showed that, despite some inaccuracies in replicating the development of volumetric strains under unsaturated conditions, the model predictions agree satisfactorily with the available measurements. Most notably, the simulations illustrated that by constraining the model on the basis of the measured data scatter, it was possible to properly replicate the transition from a contractive to dilative behavior upon shearing observed for unsaturated samples. Future investigations will be required to better quantify the influence of suction on 

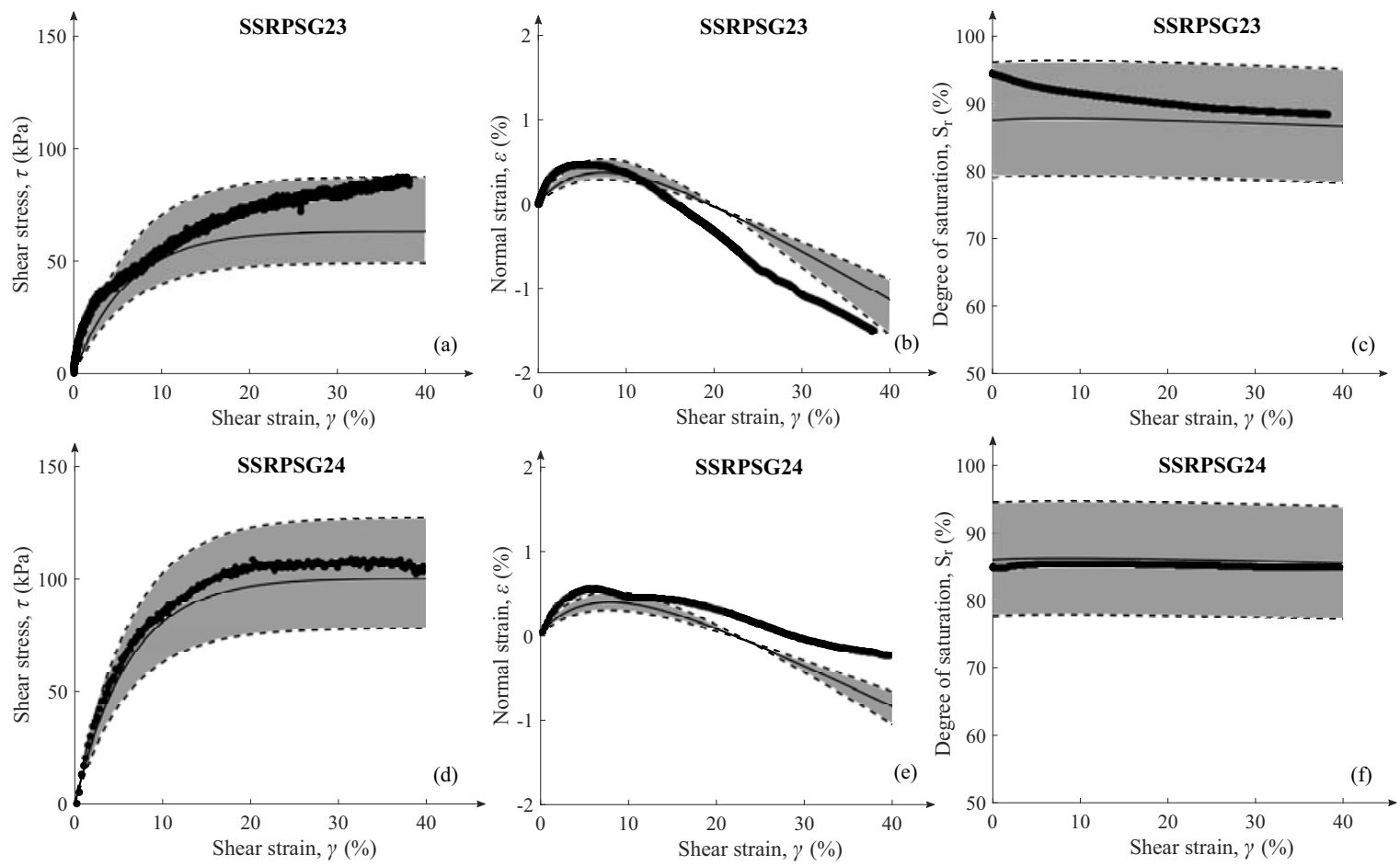

Figure 8. Simulations of SS tests under unsaturated conditions:(a) $\gamma-\tau$ response of test ID. SSRPSG23; (b) $\gamma-\varepsilon$ response of test ID. SSRPSG23; (c) $\gamma-S_{r}$ response of test ID. SSRPSG23; (d) $\gamma-\tau$ response of test ID. SSRPSG24; (e) $\gamma-\varepsilon$ response of test ID. SSRPSG24 (f) $\gamma-S_{r}$ response of test ID. SSRPSG24.

the failure envelope and verify the model performance for wetting tests. Moreover, a further development may involve an explicit quantification of the agreement between data and computations based on automatic parameter optimization algorithms.

\section{Acknowledgements}

Giuseppe Buscarnera gratefully acknowledges the financial support of the U.S. National Science Foundation through Grant No. CMMI-1351534.

\section{References}

[1] J. Wartman, D.R. Montgomery, S.A. Anderson, J.R. Keaton, J. Benoît, J.and dela Chapelle, R. Gilbert, Geomorphology 253, 275 (2016)

[2] L. Cascini, S. Cuomo, D. Guida, Engineering Geology 96, 107 (2008)

[3] J. Lizárraga, G. Buscarnera, Géotechnique 68, 442 (2017)

[4] J.J. Lizárraga, P. Frattini, G.B. Crosta, G. Buscarnera, Engineering geology 228, 346 (2017)

[5] S. Cuomo, M. Moscariello, V. Foresta, Géotechnique Letters 7, 197 (2017)

[6] M. Moscariello, Ph.D. thesis, University of Salerno, p 233 (2017)

[7] A.W. Bishop, Teknisk ukeblad 39, 859 (1959)
[8] D. Sun, D. Sheng, S.W. Sloan, Mechanics of Materials 39, 212 (2007)

[9] E.E. Alonso, J.M. Pereira, J. Vaunat, S. Olivella, Géotechnique 60, 913 (2010)

[10] M.T. Van Genuchten, Soil science society of America journal 44, 892 (1980)

[11] J.J. Lizárraga, G. Buscarnera, PanAm Unsaturated Soils 2017 (2018)

[12] K. Been, M.G. Jefferies, Géotechnique 35, 99 (1985)

[13] X.S. Li, Y.F. Dafalias, Geotechnique 50, 449 (2000)

[14] R. Lagioia, A.M. Puzrin, D.M. Potts, Computers and Geotechnics 19, 171 (1996)

[15] D. Gallipoli, A. Gens, R. Sharma, J. Vaunat, Géotechnique. 53, 123 (2003)

[16] M. Moscariello, S. Cuomo, F. Vito, Proceedings of 7th International Conference on Unsaturated Soils (2018)

[17] E. Bilotta, L. Cascini, V. Foresta, G. Sorbinow, Geotechnical \& Geological Engineering 23, 365 (2005)

[18] E. Bilotta, V. Foresta, G. Migliaro, 1, 349 (2008)

[19] C. Mihalache, G. Buscarnera, Journal of Engineering Mechanics 142, 04016091 (2016)

[20] G. Buscarnera, C. Di Prisco, Géotechnique Letters 1, 85 (2011)

[21] G. Buscarnera, C. Di Prisco, Géotechnique 63, 801 (2013) 